\title{
Cox1 barcoding versus multilocus species delimitation: validation of two mite species with contrasting effective population sizes
}

\author{
Pavel B. Klimov ${ }^{1,2^{*}} \mathbb{D}$, Maciej Skoracki ${ }^{3}$ and Andre V. Bochkov ${ }^{2,4^{\wedge}}$
}

\begin{abstract}
Background: The cox1-barcoding approach is currently extensively used for high-throughput species delimitation and discovery. However, this method has several limitations, particularly when organisms have large effective population sizes. Paradoxically, most common, abundant, and widely distributed species may be misclassified by this technique.

Results: We conducted species delimitation analyses for two host-specific lineages of scab mites of the genus Caparinia, having small population sizes. Cox1 divergence between these lineages was high (7.4-7.8\%) while that of nuclear genes was low (0.06-0.53\%). This system was contrasted with the medically important American house dust mite, Dermatophagoides farinae, a globally distributed species with very large population size. This species has two distinct, sympatric cox 1 lineages with 4.2\% divergence. We tested several species delimitation algorithms PTP, GMYC, ABGD, BPP, STACEY and PHRAPL, which inferred different species boundaries for these entities. Notably, STACEY recovered the Caparinia lineages as two species and D. farinae as a single species. BPP agreed with these results when the prior on ancestral effective population sizes was set to expected values, although delimitation of Caparinia was still equivocal. No other cox 1 species delimitation algorithms inferred D. farinae as a single species, despite the fact that the nuclear CPW2 gene shows some evidence for introgression between the cox1 groups. This indicates that the cox1-barcoding approach may result in excessive species splitting.

Conclusions: Our research highlights the importance of using nuclear genes and demographic characteristics to infer species boundaries rather than relying on a single-gene barcoding approach, particularly for putative species having large effective population sizes.
\end{abstract}

Keywords: Species delimitation, cox1, Barcoding, Large population size, Mito-nuclear discordance

\section{Background}

The DNA barcoding approach is a useful tool for DNA-based, automatic identification of organisms. Because this approach relies on sequencing of a standardized gene region, the "barcode", a specimen can be identified by comparing its sequence to a reference database [1, 2], for example, GenBank or BOLD [3]. Typically, for animals, the standard locus is the Folmer fragment of the

\footnotetext{
* Correspondence: pklimov@umich.edu

Deceased

${ }^{1}$ Department of Ecology and Evolutionary Biology, Museum of Zoology, University of Michigan, 3600 Varsity Drive, Ann Arbor, Michigan 48108, USA

${ }^{2}$ Tyumen State University, 10 Semakova Str, 625003 Tyumen, Russia

Full list of author information is available at the end of the article
}

mitochondrial gene, cytochrome $c$ oxidase subunit 1 ( $\operatorname{cox} 1)$ [2], for fungi it is ITS2 [4], while for plants, two loci from the plastid genome are used [5]. To be successful, a DNA barcoding approach should meet three basic criteria: (i) a sufficient amount of variation exists in the barcode region to distinguish species; (ii) no overlap between intra- and inter-specific genetic distances; and (iii) a prior knowledge of species boundaries. Here, the notion of a barcoding gap, a "break" in the distribution among withinand between-species variation distances, is very important. In practice barcoding gap analyses are widely used for species delimitation, assigning specimens to species when species boundaries are unknown, often in conjunction with building a phylogenetic or distance-based tree [6, 7]. In many cases, no single threshold or barcoding gap exist 
that can be used to assign all specimens without incurring high error rates [7-10]. Typical barcoding gap values (Kimura 2-parameter genetic distances, K2P) range between $\sim 2$ to $4 \%$, above which genetic distances are considered to be interspecific $[3,6,10-13]$. These values can be either used as predetermined thresholds [11] or, more appropriately, as useful prior threshold values in automatic gap discovery analyses [14]. However, some species, particularly those having large population sizes, show maximum within-species cox1 distances much higher than these values: $15.4 \%$ in the Chinese perch Siniperca chuatsi [10]; $10.1 \%$ in the human follicle mite Demodex folliculorum (Demodecidae) (conservatively recalculated from [15]); $5.7-6.8 \%$ in the common blue butterfly Polyommatus icarus (Lycaenidae) [16]; about $6 \%$ in the sea snail Echinolittorina vidua (Littorinidae) [17]; 4.3\% in the mold mite Tyrophagus putrescentiae [18]; and $4.2 \%$ in the American house dust mite (our data) to name a few. Cox 1 barcoding performs well when species have small population sizes, low speciation rates [19] or substantial divergence times [10]. Thus, paradoxically, most common and widely distributed species, such as those listed above, are in the 'gray zone' of the cox 1 barcoding approach and may present methodological challenges for the DNA barcoding approach.

Population genetic theory-based alternatives to threshold-based approaches can accurately delimit species under a range of conditions, including variable population sizes and times of origins $[8,20]$. Two recently proposed species delimitation methods, BPP [21] and STACEY [22], are both based on the multispecies coalescent model and assume that species are distinct populations without gene flow. The latter is estimated by taking into account the ancestral population size and time of divergence at the root, while species trees are estimated under a coalescent process, assuming neutral evolution and no selection for single or multiple loci. When all these parameters are estimated (or fixed to a known value), posterior probabilities for alternative species delimitation models can be calculated, and the best-fitting model can be selected objectively. Another species delimitation approach that uses multispecies coalescent, PHRAPL [23], is based on a likelihood framework and, in addition, also incorporates gene flow when estimating species boundaries. The disadvantages of these methods are: (i) the need to estimate population genetic parameters that are typically unknown (except for PHRAPL, which estimates them using Maximum Likelihood); (ii) use of phased sequences of nuclear loci (i.e. polymorphisms in sequences should be phased out to represent the two alleles of a diploid organism); (iii) $a$ priori specimen assignment to a 'minimal' population in several cases; and (iv) the assumption of neutral evolution. In addition, multispecies coalescent methods can be computationally prohibitive and are only feasible for small sets of species with unclear boundaries. Despite being methodologically superior, multispecies coalescent methods have their own 'gray zone' where conflicting species delimitations are possible - typically, when gene trees have shallow branch lengths (recent speciation events) and when lineages have small effective population sizes (higher probability of speciation due to drift).

Here we explore several methods of species delimitations, the threshold-based ABGD [14], the multispecies coalescent-based BPP, STACEY and PHRAPL, as well as other algorithms, GMYC [24] and PTP [25]. Our specific goal was to evaluate the species status of mostly host-specific populations of scab mites of the genus Caparinia (family Psoroptidae) parasitizing two species of hedgehogs, the European hedgehog Erinaceus europaeus and the African hedgehog Atelerix albiventris [26-30]; the latter species being a popular pet throughout the world. K2P cox1 distances between the two populations were 7.48-7.77\% (our data). These mites are rare in the field (our data; Additional file 1: Text S1), suggesting that their population sizes are relatively small. Despite the large $\operatorname{cox} 1$ distances between these populations, nuclear genes of these lineages show only minimal variation (0.09-0.53\%; our data, see below). Phenotypic differences were also minimal and do not allow clear-cut taxonomic judgment on whether these populations are either a single or separate species [31, 32]. Therefore, our model system allows testing whether distinct cox1-based clades are sufficient to delimit species when nuclear genes form shallow clades and phenotypic differences between lineages are minimal, which might suggest a recent divergence event between these lineages and, therefore, rapid speciation rates. Thus, our empirical system may be in the 'gray zone' of molecular taxonomy. For comparative purposes, we also employ another model system, the American house dust mite Dermatophagoides farinae, which is a globally distributed species with a large population size. It has a strongly structured population with two cox 1 lineages having a $4.19 \%$ K2P divergence. To calculate a barcoding gap without potential influence of technical errors or removing the $5 \%$ "outliers" [9,33], we employ a well-curated cox 1 sequence database (Additional file 2: Table S1), including two closely related families, the psoroptic scab mites (Psoroptidae) and pyroglyphid house dust mites (Pyroglyphidae). These families contain cosmopolitan, free-living species with large effective population sizes (house dust mites Dermatophagoides farinae and $D$. pteronyssinus), and either multiple- (Psoroptes ovis, Chorioptes bovis) or single-host (Choirioptes sweatmani) parasites. 


\section{Results}

\section{Quality of GenBank data}

Out of 12 pyroglyphid cox 1 GenBank sequences (Additional file 3: Figure S1), 10 (83.3\%) were excluded: Dermatophagoides farinae China (KP871846.1-KP87185 0.1, KX211988.1-KX211990.1; unusual amino acid substitutions); Dermatophagoides pteronyssinus Thailand (HQ823623.1; unusual amino acid substitutions, stop codons, and frameshifting insertions); Dermatophagoides farinae Thailand (HQ823622.1; unusual amino acid substitutions, stop codons, and frameshifting insertions). Only two sequences (16.7\%) passed our quality filter criterion: Dermatophagoides pteronyssinus Belgium (EU884425.1) and Euroglyphus maynei USA (MUJZ0107 2749.1; annotated alignment in Additional file 4). Low quality sequences tend to occupy basal positions within species subclades, e.g. groups 1 and 2 of Dermatophagoides farinae, creating a false impression of their earlier origins (Additional file 3: Figure S1). After removal of the suspect sequences, minimunmaximum K2P cox1 genetic distances changed only marginally: Dermatophagoides microceras vs D. farinae (9.34-10.02 vs 9.00-10.22\% before the removal); $D$. farinae vs D.farinae (maximum of 4.19 vs $4.57 \%$ before the removal); $D$. pteronyssinus vs $D$. pteronyssinus (maximum of 1.97 vs $2.14 \%$ before the removal).

\section{Morphological differences}

We found the following differences between Caparinia tripilis versus mites from Atelerix albiventris and Ictonyx striatus (hereafter referred to as Caparinia ictonyctis, see the Discussion section). In females of C. ictonyctis, setae si are situated off the small plates bearing setae se (Fig. 1a), while in C. tripilis these setae are on or, more rarely, off, the small plates (Fig. 1b). In males of C. ictonyctis, coxal fields III are completely closed (Fig. 1c), while in C. tripilis, coxal fields III are semienclosed (Fig. 1d).

\section{Genetic distances}

To calculate a barcoding gap without potential influence of technical errors or removing the 5\% "outliers", we employed a well-curated cox 1 sequence database, including two closely related families, the psoroptic scab mites (Psoroptidae) and pyroglyphid house dust mites (Pyroglyphidae).

Among the seven loci, the mitochondrial proteincoding gene cox 1 had the largest within- and among-species distances $(0-6.0 \%$ and $4.3-15.5 \%$, respectively) (Fig. 2, Additional file 5: Table S2). Nuclear genes with the highest between-species K2P distances were SRP54 (0.2-8.0\%) and HSP70 (0.2$7.9 \%$ ), while $18 \mathrm{~S}$ had the lowest genetic distances

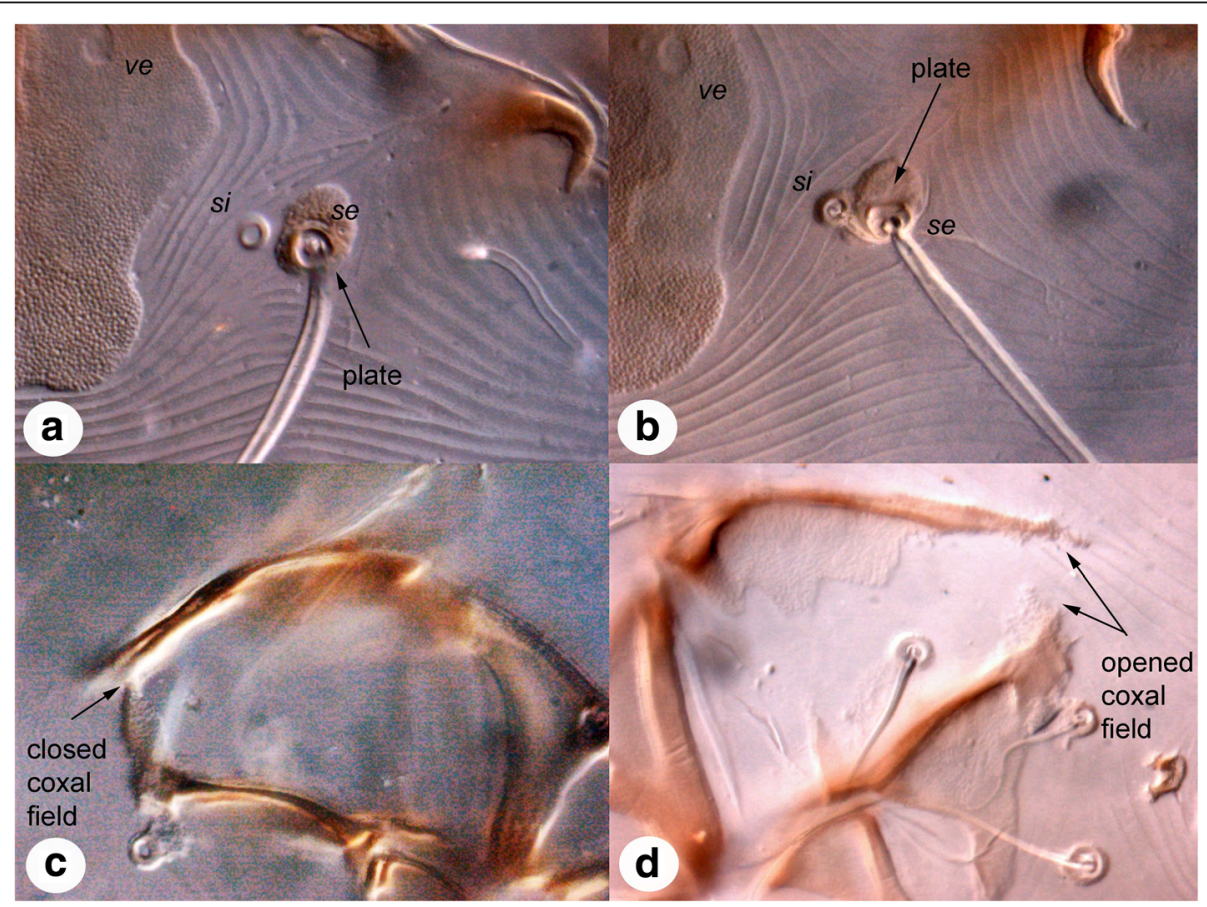

Fig. 1 Diagnostic characters of Caparinia tripilis and C. ictonyctis. a Position of seta si, female of C. ictonyctis stat. res. b Position of seta si, female of C. tripilis; c Coxal field III, male of C. ictonyctis stat. res. d Coxal field III, male of C. tripilis 


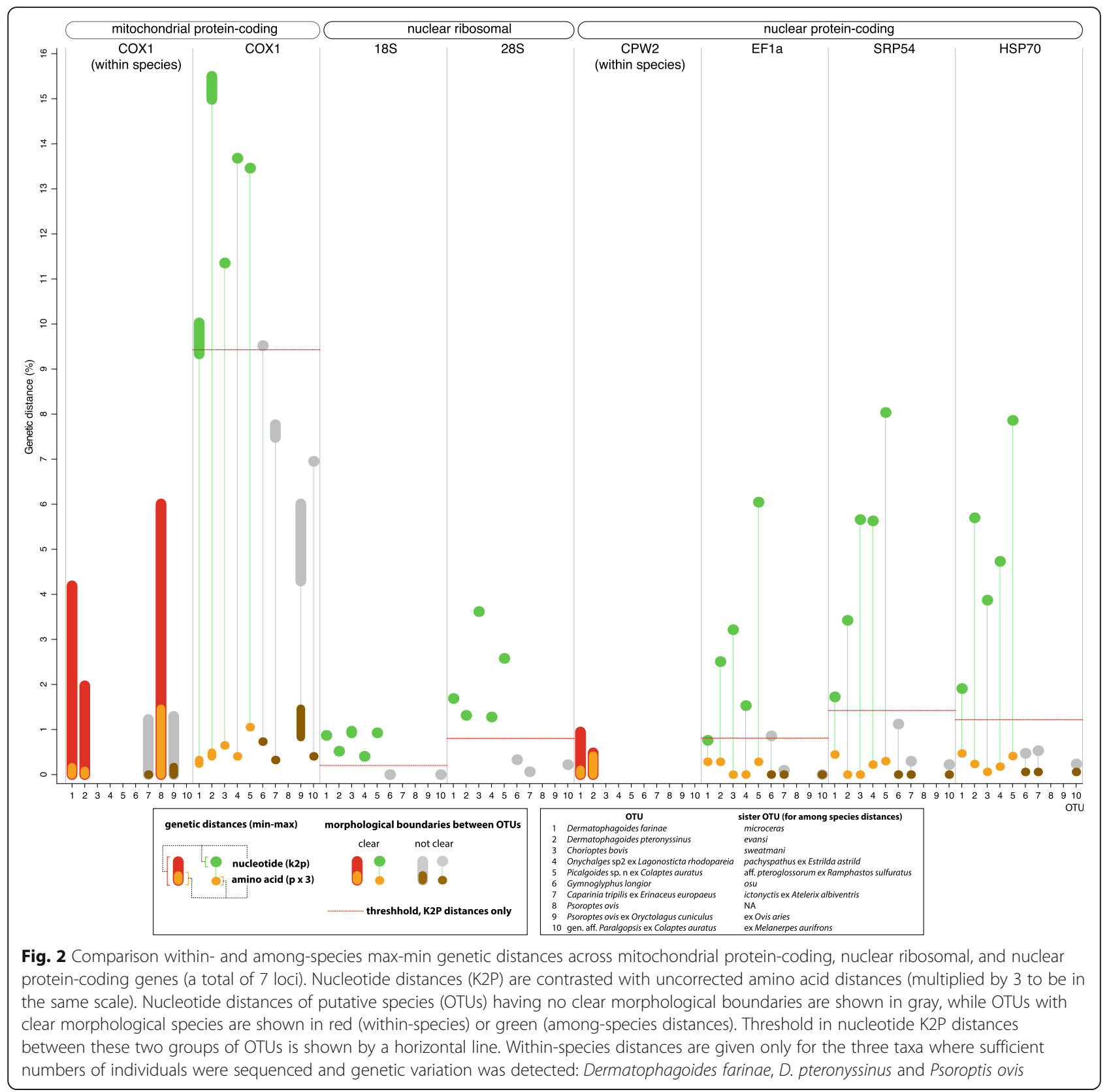

(0-1.0\%) (Fig. 2, Additional file 5: Table S2). For nuclear genes, within-species distances were available only for CPW2: 0-0.95\% (Dermatophagoides farinae) and $0-0.48 \%$ (D. pteronyssinus) (Additional file 6: Figure S2: contract of cox1 vs CPW2 phylogenies).

There was no clear threshold between within- and between-species $\operatorname{cox} 1$ distances, given the fact that putative species with no clear morphological differences may be or may not be true species (Fig. 2; shown by gray) or may represent two or more true species (e.g. Psoroptis ovis). Nevertheless, for cox1, a 'conservative' threshold of $>9.52 \%$, e.g. $9.6-10 \%$ in K2P distances, could distinguish all 'good' species, i.e. those having clear morphological differences (Fig. 2).

If the extreme value of $C P W 2$ within-species distances $(0.95 \%)$ is taken as an 'universal' species cut-off for other nuclear genes, then misclassifications will occur for OTUs with no clear morphological differences for all genes (Table 1; compare $0.95 \%$ with minimum values; Fig. 2 ). For OTUs with clear morphological differences, misclassifications will occur in two loci, $E F 1-\alpha$ and $18 S$, which have minimum between-species distances below this threshold (Table 1, Fig. 2). It is notable, that in D. pteronyssinus, $C P W 2$ is probably under a strong selection because the 
Table 1 Comparison of genetic distances (K2P) between two groups of putative species: with and without clear morphological differences

\begin{tabular}{|c|c|c|c|c|c|c|}
\hline \multirow[t]{3}{*}{ Locus } & \multicolumn{6}{|c|}{ Morphological differences between OTUs } \\
\hline & \multicolumn{2}{|l|}{ Clear } & \multicolumn{2}{|c|}{ Not clear } & \multirow[b]{2}{*}{ Gap } & \multirow[b]{2}{*}{ Threshold } \\
\hline & Min & Max & Min & Max & & \\
\hline $\operatorname{cox} 1$ & 9.3369 & 15.4977 & 4.2953 & 9.5194 & -0.1825 & $9.4281(9.5194)$ \\
\hline$P 5$ & 1.7246 & 8.0358 & 0.2226 & 1.1215 & 0.6031 & 1.4230 \\
\hline HSP70 & 1.9088 & 7.8612 & 0.2352 & 0.5301 & 1.3787 & 1.2194 \\
\hline $1-a$ & 0.7607 & 6.0463 & 0.0000 & 0.8577 & -0.0969 & $0.8092(0.8577)$ \\
\hline 285 & 1.2775 & 3.6173 & 0.0632 & 0.3302 & 0.9473 & 0.8039 \\
\hline 185 & 0.4043 & 0.9641 & 0.0000 & 0.0000 & 0.4043 & 0.2022 \\
\hline
\end{tabular}

Abbreviations: Min, minimum; Max, maximum

ratio of synonymous $v s$ non-synonymous mutations is very high (Fig. 2).

Even though it was not possible to establish a universal species delimitation gap for nuclear genes, most loci (SPR54, HSP70, 28S, 18S) have a clear K2P gap between putative species with and without clear morphological differences (Table 1, Fig. 2), although distances for cox 1 and EF1- $\alpha$ slightly overlapped (Table 1, Fig. 2).

Amino acid distances lack a clear threshold-like pattern allowing distinguishing either among putative or 'good' species (Additional file 5: Table S2, Fig. 2). For example, 'good' species Chorioptes bovis and Ch. sweatmani lack any amino acid substitutions for EF1- $\alpha$ and SPR54, while HSP70 had only a single substitution.

\section{Species delimitation \\ GMYC}

Analyses using trees inferred under different speciation models (i.e. Yule $v s$ coalescent) and molecular evolution (i.e. relaxed $v s$ strict clock) resulted in the same species delimitation scheme containing 49 species and nearly the same threshold times, -0.0131 to -0.0126 (Additional file 7 : Table S3: columns 5-6). This scheme was exactly the same as the one found by the PTP Maximum Likelihood and $\mathrm{ABGD}(\mathrm{X} 1=1.1, \mathrm{P}=1.29 \%)$ analysis (see below).

PTP

The Maximum Likelihood solution had 49 species, which was exactly the same found by GMYC (see above) and ABGD with $\mathrm{X}=1.1$ (see below), where Caparinia, Dermatophagoides farinae and Psoroptes ovis were each split into two separate species (Additional file 7: Table S3: columns 1-2). The Bayesian solution had 52 species; the difference was due to excessive oversplitting of Psoroptes ovis ex Ovis aries and Dermatophagoides farinae group 1 (Additional file 7: Table S3: columns 3-4).

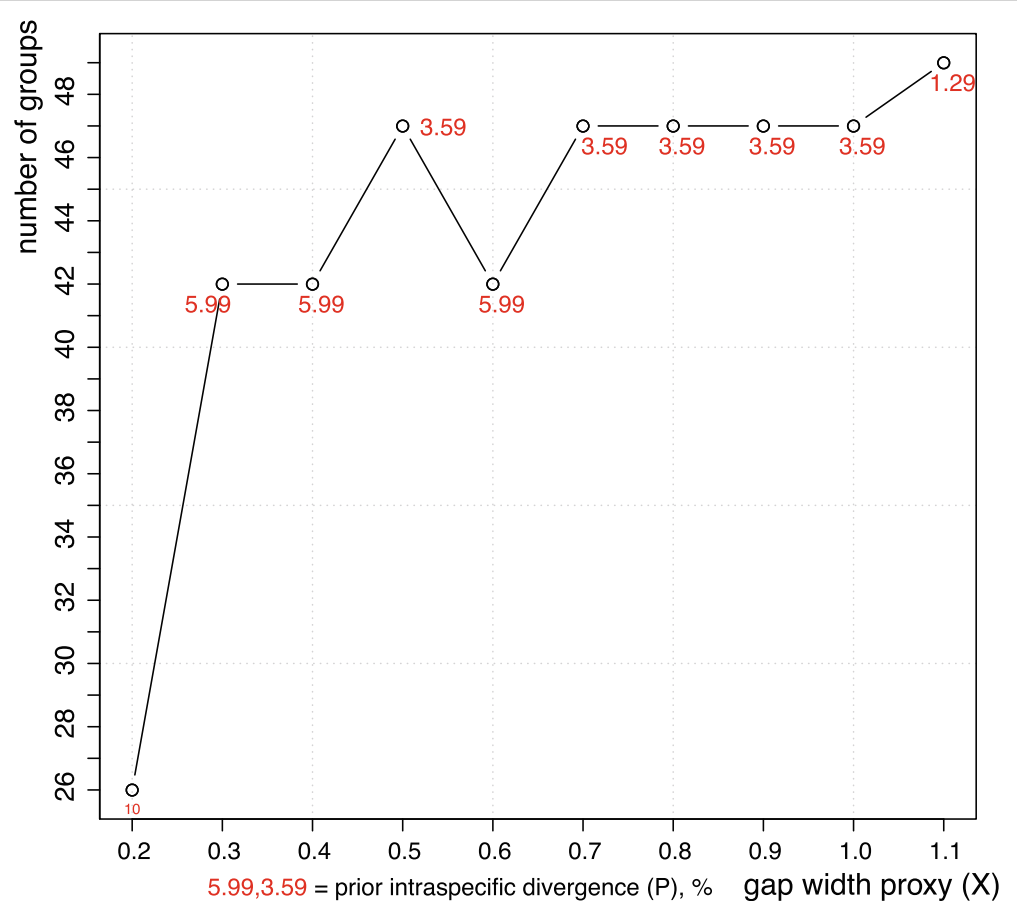

Fig. 3 Summary of $10 \mathrm{ABGD}$ runs with the gap width proxy parameter varied in the range from 0.2 to 1.1. Number of groups (putative species) recovered by the last multispecies $A B G D$ iteration as well as the barcoding gap value are reported 


\section{$A B G D$}

The highest possible value of the barcoding gap width proxy parameter $(\mathrm{X}=1.1)$ gave a 49 -species delimitation (Fig. 3), exactly the same as the PTP Maximum Likelihood and GMYC solutions (Additional file 7: Table S3: columns 7-8). A range of lower values ( $\mathrm{X}=1.0-0.7$ and 0.5 ) resulted in a 47 -species scenario where $D$. farinae and Psoroptes ovis were each a single species, but the two Caparinia OTUs were still two separate species (Additional file 7: Table S3: columns 9-10). Lower values of $\mathrm{X}(\mathrm{X}=0.6$ and 0.4$)$ yielded a 42 -species delimitation (Additional file 7: Table S3: columns 11-12). Notably, all "gray zone" taxon pairs (weak or no morphological differences) were collapsed (Fig. 2, Additional file 7: Table S3: columns 7-12). In addition, Microlichus sp. ex Hirundo rustica (Russia) and Microlichus sp. ex Amazilia tzacatl (Mexico) were collapsed to a single species; and Dermatophagoides microceras was collapsed with Dermatophagoides farinae (closely related species having distinct shapes of the female spermatheca). Setting the barcoding gap width proxy to $\mathrm{X}=0.2$ resulted in a 26-species delimitation scheme (Fig. 3). Many well-recognized species from different genera or families were collapsed to a single one. For example, Picalgoides spp., Mesalgoides spp., Paralgopsis spp. and Onychalges spp. were recovered as a single species (Additional file 7: Table S3: columns 13-14). Because of a major decrease of sensitivity of the method with $\mathrm{X}=0.2$, no further analyses were performed. Prior intraspecific divergence was strictly negatively correlated with the number species recovered (Fig. 3): $1.29 \%=49$ species; $3.59 \%=47$ species; $5.99 \%=42$ species; and $10 \%=26$ species (Fig. 3). Notice that these values represent a prior intraspecific divergence, which is used by the program to find a barcoding gap above the given value.

\section{BPP}

For the Caparinia dataset, analyses with the three sets of priors, reflecting different ancestral population sizes $(\theta)$ and root ages $(\mathrm{\tau} 0)$, all inferred a two-species model, lumping Caparinia ictonyctis from Atelerix albiventris together with $C$. tripilis from Erinaceus europaeus into a single species (Table 2). Posterior support for this model was moderate $(0.863,0.787)$, or low (0.514) for the model assuming both small population sizes at root and root age (Table 2). All analyses suggest a large decrease, 90.13-93.20\%, of effective population size at the divergence of the two Caparinia OTUs (Table 2). For the Dermatophagoides dataset, analyses using the three sets of population genetics priors differed in whether Dermatophagoides farinae OTUs, Dfa and DFb, are a single or two separate species. When ancestral population size $(\theta)$ and root ages $(\tau)$ are large then these two OTUs are recovered as a single species with high probability $[\mathrm{PP}=0.9537$ (model), $\mathrm{PP}=0.9886$ (species) $]$, while analyses with other priors suggest that these two mitochondrial-only groupings are separate species, with weak support for the 4-OTU species delimitation model + topology $(\mathrm{PP}=0.5428,0.5917$; Table 2). However, posterior probabilities for the two OTUs (DFa, DFb) being separate species were high, 1.00.9585 and 0.9990-0.9480, respectively (Table 2).

\section{STACEY}

For the Caparinia dataset, the model treating the two host-specific Caparinia lineages as different species had a better relative fit than the model treating these lineages as a single species. Marginal likelihoods for these models were $-16156.3 \pm 0.173$ vs $-16161.8 \pm$ 0.161 , respectively (mean $\pm \mathrm{SE}$ ). The difference was $\mathrm{BF}=5.56$, suggesting that there is positive evidence for the two Caparinia species: C. tripilis and C. ictonyctis. For the Dermatophagoides dataset, an analysis where the two groups of Dermatophagoides farinae (Dfa and DFb) were merged into a single species ("minimal cluster") had a better relative fit than the species delimitation model treating these two groups as two distinct species. Marginal likelihoods for these models were $($ mean $\pm \mathrm{SE}):-5932.4 \pm 0.14$ vs -5935.5 \pm 0.36 , respectively. The difference was $\mathrm{BF}=3.01$, suggesting that there is positive evidence for the model treating Dermatophagoides farinae as a single species. Similarly, a STACEY species discovery analysis grouped the two $D$. farinae groups into a single species (Additional file 8: Figure S3).

\section{PHRAPL}

For Dermatophagoides farinae, among the nine PHRAPL models with $\triangle \mathrm{AIC}$ less than 2, all were 3and 2-species models (Additional file 9: Table S1). The best model (AIC 54.53) was a 3-species, isolation-only model (no gene flow), the second best model (AIC 55.47) was a 3-species, isolation + migration model, with two symmetrical migration rates: clades 1 and ancestral clade $2+3$, and clades 2 and 3 . The third best-scoring model (AIC 55.49) was a 2 -species, isolation-only model, where clades 2 and 3 were collapsed. In all these models, gdi scores for clade $1+$ ancestror for clades $2+3$ (i.e. basal dichotomy of Dermatophagoides farinae) were high $(0.994,0.999$ and 0.995 , respectively); while gdi scores for clades $2+3$ were medium or high $(0.524,0.953$ and 0.524 , respectively). The best-fitting 1-species model was a migration-only model $(\mathrm{dAIC}=6.83$, gdi $=0.001)$. 
Table 2 Summary of BPP species delimitation analyses of Caparinia (5 loci) and Dermatophagoides (2 loci) datasets using three sets of priors for ancestral population size ( $\theta$ ) and root age (T0). Parameter estimates (means, 2.5-97.5\% HPD intervals), posterior probabilities (PP) for select species delimitation models and OTUs are given

\begin{tabular}{|c|c|c|c|c|c|c|c|}
\hline \multirow[t]{3}{*}{ Species } & \multirow[t]{3}{*}{ Species tree/Pr } & \multicolumn{6}{|c|}{ Prior distributions } \\
\hline & & \multicolumn{2}{|c|}{$\begin{array}{l}\theta \sim \mathrm{G}(1,10) \\
\mathrm{T} 0 \sim \mathrm{G}(1,10)\end{array}$} & \multicolumn{2}{|c|}{$\begin{array}{l}\theta \sim \mathrm{G}(2,1000) \\
\mathrm{T} 0 \sim \mathrm{G}(2,1000)\end{array}$} & \multicolumn{2}{|c|}{$\begin{array}{l}\theta \sim \mathrm{G}(1,10) \\
\mathrm{T} 0 \sim \mathrm{G}(2,1000)\end{array}$} \\
\hline & & $\mathrm{PP} /$ mean & Sampled HPD & $\mathrm{PP} /$ mean & Sampled HPD & $\mathrm{PP} /$ mean & Sampled HPD \\
\hline & Caparinia & & & & & & \\
\hline & $\theta($ root $)$ & 0.1851 & $0.0776-0.3245$ & 0.0304 & $0.0229-0.0383$ & 0.1867 & $0.0775-0.3227$ \\
\hline & $\theta($ Caic,Catri) & 0.0167 & $0.0004-0.0437$ & 0.003 & $0.0004-0.0061$ & 0.0127 & $0.0004-0.0343$ \\
\hline & $\tau($ root $)$ & 0.0037 & $0.0002-0.0075$ & 0.0015 & $0.0002-0.0031$ & 0.0024 & $0.0003-0.0043$ \\
\hline & $\mathrm{T}($ Caic,Catri) & 0.0003 & $0-0.0008$ & 0.0005 & $0-0.001$ & 0.0003 & $0-0.0008$ \\
\hline 2 & (Ocy, CaicCatri) & 0.86285 & 86,285 & 0.51417 & 51,417 & 0.78684 & 78,684 \\
\hline 3 & (Ocy, (Caic, Catri)) & 0.13035 & 13,035 & 0.48054 & 48,054 & 0.21061 & 21,061 \\
\hline \multirow[t]{11}{*}{1} & (OcyCaicCatri) & 0.0068 & 680 & na & na & 0.00237 & 237 \\
\hline & $\operatorname{Pr}($ Ocy) & 0.9932 & 99,320 & 0.999 & 99,900 & 0.99763 & 99,763 \\
\hline & $\operatorname{Pr}($ CaicCatri) & 0.86285 & 86,285 & 0.51417 & 51,417 & 0.78684 & 78,684 \\
\hline & $\operatorname{Pr}($ Caic) & 0.13035 & 13,035 & 0.48531 & 48,531 & 0.21079 & 21,079 \\
\hline & $\operatorname{Pr}($ Catri) & 0.13035 & 13,035 & 0.48535 & 48,535 & 0.21079 & 21,079 \\
\hline & $\operatorname{Pr}($ OcyCaicCatri) & 0.0068 & 680 & na & na & 0.00237 & 237 \\
\hline & Dermatophagoides & & & & & & \\
\hline & $\theta($ root $)$ & 0.2105 & $0.0193-0.4115$ & 0.0341 & $0.0262-0.0425$ & 0.2297 & $0.095-0.3978$ \\
\hline & $\theta(\mathrm{DFa}, \mathrm{DFb})$ & 0.0407 & $0.0051-0.0954$ & 0.0037 & $0.0016-0.0069$ & 0.0048 & $0.0021-0.0087$ \\
\hline & $\tau($ root $)$ & 0.0141 & $0.0029-0.0335$ & 0.0050 & $0.0018-0.0085$ & 0.0107 & $0.0009-0.0258$ \\
\hline & $\mathrm{t}(\mathrm{DFa}, \mathrm{DFb})$ & 0.0004 & $0-0.001$ & 0.0003 & $0-0.0008$ & 0.0003 & $0-0.0008$ \\
\hline 4 & $(\mathrm{DP},(\mathrm{DM},(\mathrm{DFa}, \mathrm{DFb})))$ & 0.0104 & 1042 & 0.5428 & 54,284 & 0.5917 & 59,174 \\
\hline \multirow[t]{4}{*}{3} & $(\mathrm{DP},(\mathrm{DM}, \mathrm{DFaDFb}))$ & 0.9537 & 95,373 & na & na & 0.0266 & 2661 \\
\hline & $\operatorname{Pr}(\mathrm{DFa})$ & 0.0115 & 1145 & 1.0000 & 100000 & 0.9585 & 95,845 \\
\hline & $\operatorname{Pr}(\mathrm{DFb})$ & 0.0115 & 1145 & 0.9990 & 99903 & 0.9480 & 94,802 \\
\hline & $\operatorname{Pr}(\mathrm{DFaDFb})$ & 0.9886 & 98,855 & na & na & 0.0416 & 4155 \\
\hline
\end{tabular}

Abbreviation: na, not available

\section{Discussion}

Morphological discontinuities, genetic distances, and species delimitation

Even though using predetermined thresholds for species delimitation quickly falls into disrepute, the knowledge of approximate values separating withinversus between species genetic distances is still important. For example, it can be used to filter out suspect sequences (misidentifications, sequencing artifacts) from public databases $[9,33]$ or as a starting point (prior) in automatic gap discovery analyses [14]. Misspecification of this prior may result in inaccuracies in species delimitation by this method. Based on our curated Pyroglyphidae + Psoroptidae dataset, a 'conservative' distance of $>9.52 \% \mathrm{~K} 2 \mathrm{P}$ distance was able to distinguish species that have clear morphological differences (Table 1). This value is very close to the average smallest interspecific distances (9\%) reported for feather mites [34]. Below the 9.52\% 'conservative' distance there was a "gray" species delimitation zone, where OTUs could not be unambiguously assigned to species based on morphology. It is notable that our 'conservative' cox 1 threshold is much higher than values used in literature (4\% [11], 3.14\% [34], 3\% [6, 12], 2\% [7, 12, 13], or lower [6]). Applying even the highest of these threshold values to our dataset will split species having large, strongly structured and presumably panmictic populations. For example, in the American house dust mite, Dermatophagoides farinae, cox 1 suggests the existence of two distinct groups, 1 and 2 (Additional file 6: Figure S2) having a maximum $\mathrm{K} 2 \mathrm{P}$ distance of $4.2 \%$. However, the nuclear CPW2 gene did not support these cox1-only groupings (Additional file 6: Figure S2), 
suggesting that, while some population structure does exist, members of different lineages are likely to interbreed (as evidenced by CPW2 polymorphic individuals), and there is gene flow between them. Alternative explanation for this pattern is very recent lineage divergence. Similarly, Psoroptes ovis, a parasitic scab mite known from a wide range of domesticated and wild animals, forms two sister groups clearly separated by the nuclear ITS locus and microsatellites [35-38]. These groups are not host-specific and do not have clear morphological differences [36, 39]; one of them, the minority group, probably corresponds to our 'rabbit' group $(\operatorname{cox} 1 \mathrm{~K} 2 \mathrm{P}=6.0 \%)$. Given our results, we believe that OTUs delimited by cox 1 genetic distances lower than $9.52 \%$ need to be corroborated by independent lines of evidence, such as sequences of nuclear genes or breeding experiments for sexual species, rather than taken as conclusive evidence for the presence of distinct species. In contrast to cox 1 , nuclear genes showed variable thresholds from 0.2 to $1.4 \%$, with SPR54 and HSP70 thresholds being the highest, and $18 S$ being the lowest (Table 1).

\section{cox 1 barcode species delimitation}

There was a total of 42-49 plausible species delimitation schemes based on cox1; two analyses resulted in an abnormally high (54, bPTP) or low (26, ABGD, X $=0.2, \mathrm{P}=10 \%$ ) number of species (Table 3, Fig. 4). PTP (maximum likelihood), GMYC and ABGD generally produced similar results with the maximum of 49 species. When the barcoding gap width proxy prior was set to a lower value $(\mathrm{X}<1.1)$, ABGD generally lost sensitivity, inferring 47 or 42 species. Our taxa of interest, the host-specific lineages of Caparinia, were inferred as separate species by all cox1-based analyses. Similarly, the well-behaved analyses, PTP (maximum likelihood), GMYC, and ABGD, consistently split the American house dust mite, Dermatophagoides farinae, into two species, corresponding to cox1 groups 1 and 2 (Fig. 4). However, when the $\mathrm{X}$ prior was set too low, the prior threshold was high $(\mathrm{P} \geq 5.99 \%)$ and ABGD lumped $D$. farinae and $D$. microceras. These taxa are similar but reproductively incompatible species, with clear differences in the female spermatheca [40]. Thus, unfortunately, the cox 1 analyses were not able to infer $D$. farinae within boundaries established by morphological systematics and breeding experiments (Table 3, Fig. 4).

\section{Multispecies coalescent species delimitation}

Multilocus delimitation analyses based on multispecies coalescent are computationally intensive and, therefore, were run only for our taxa of interest. For the Caparinia dataset, BPP analyses suggested lumping Caparinia ictonyctis and C. tripilis (s.s.) into a single species when both ancestral population size and root age are large $[\theta \sim G(1,10) \quad$ T0 G(1,10)] (Tables 2, 3). This, however, is an unrealistic scenario given a very low prevalence of Caparinia in natural host populations (see Additional file 1: Text S1). Under the likely set of priors, small population size and young root age $[\theta \sim G(2,1000)$ t0 G $(2,1000)]$, the single-species model was only marginally better than Caparinia being split into two host-specific species $(\mathrm{PP}=0.5142$ vs 0.4805$)$ (Table 2). Thus,

Table 3 Summary of 12 species delimitation analyses

\begin{tabular}{|c|c|c|c|c|c|}
\hline \multicolumn{2}{|c|}{ Analysis } & \multirow{2}{*}{$\begin{array}{l}\text { Loci } \\
\text { Cox1 }\end{array}$} & \multicolumn{3}{|l|}{ Number of species } \\
\hline 1 & PTP Maximum Likelihood & & $\begin{array}{l}\text { 81-taxon alignment } \\
49\end{array}$ & $\begin{array}{l}\text { Caparinia tripilis+ictonyctis } \\
(\mathrm{K} 2 \mathrm{P} \operatorname{cox} 1=7.77 \%) \\
2\end{array}$ & $\begin{array}{l}\text { Dermatophagoides farinae } \\
(\mathrm{K} 2 \mathrm{P} \operatorname{cox} 1=4.19 \%) \\
2\end{array}$ \\
\hline 2 & bPTP Highest Bayesian supported solution & $\operatorname{cox} 1$ & 54 & 2 & 6 \\
\hline 3 & GMYC (3 analyses with different trees) & $\operatorname{cox} 1$ & 49 & 2 & 2 \\
\hline 4 & $\operatorname{ABGD}(X=1.1 ; P=1.29 \%)$ & $\operatorname{cox} 1$ & 49 & 2 & 2 \\
\hline 5 & $\operatorname{ABGD}(X=1.0-0.7,0.5 ; P=3.59 \%)$ & $\operatorname{cox} 1$ & 47 & 2 & 2 \\
\hline 6 & $\operatorname{ABGD}(X=0.6,0.4 ; P=5.99 \%)$ & $\operatorname{cox} 1$ & 42 & 2 & $1+$ \\
\hline 7 & $\operatorname{ABGD}(X=0.2 ; P=10 \%)$ & $\operatorname{cox} 1$ & 26 & $1+$ & $1+$ \\
\hline 8 & BPP $[\theta \sim G(1,10) \tau 0 \sim G(1,10)]$ & $5 / 2$ loci & - & 1 & 1 \\
\hline 9 & BPP $[\theta \sim G(2,1000) \quad \tau 0 \sim G(2,1000)]$ & $5 / 2$ loci & - & $1-2$ & 2 \\
\hline 10 & BPP $[\theta \sim G(1,10)$ T0 G(2,1000)] & 5/2 loci & - & 2 & 2 \\
\hline 11 & STACEY & 5/2 loci & - & 2 & 1 \\
\hline 12 & PHRAPL & 5/2 loci & - & na & $2-3$ \\
\hline
\end{tabular}

Key: 5/2 loci, for Caparinia/Dermatophagoides datasets, respectively; 5 loci, 18S+28S, EF1-a, SRP54, HSP70, cox1 (18S and 28S were merged because they are linked): 2 loci, cox 1 and CPW2; 1+, was merged with a closest taxon

Abbreviation: na, not available 


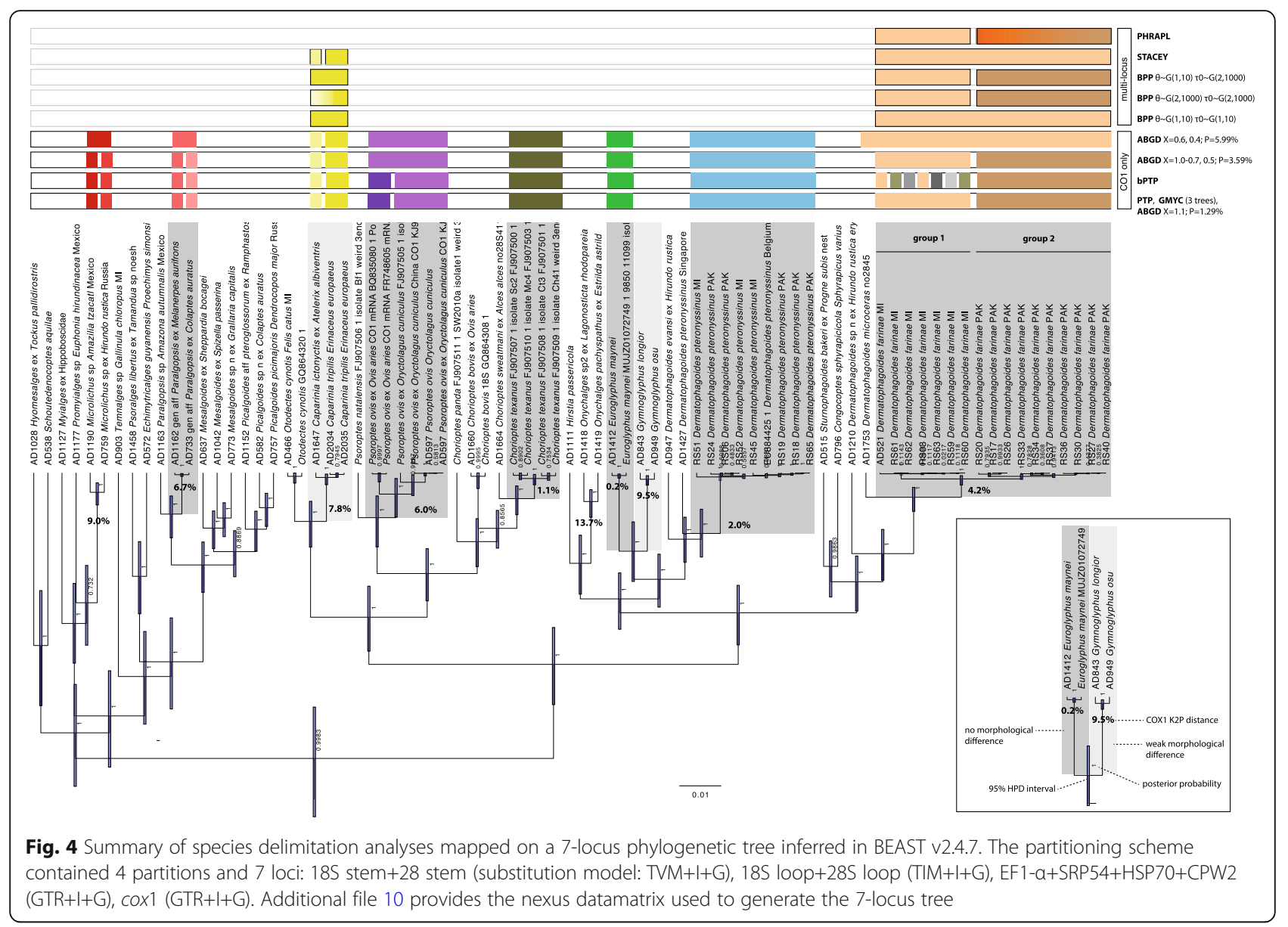

species delimitation is ambiguous here. No single solution, i.e. either one or two species, can be preferred. STACEY, another multispecies coalescent program, agrees with the two-species delimitation scheme of BPP (Table 3). BPP analyses recovered Dermatophagoides farinae as one or two species. Under realistic priors, large ancestral effective population size and old root $[\theta \sim \mathrm{G}(1,10)$ т0 G(1,10)], a single-species scenario was preferred (Table 2). STACEY agreed with this delimitation. Surprisingly, PHRAPL did not recover this scenario within a set of top-ranking delimitation models ( $\triangle \mathrm{AIC}$ range $0-2$ ), with the best-fitting single-species model having a $\triangle \mathrm{AIC}$ of 6.83 (Additional file 9: Table S1). This program extensively relies on "testing" species delimitation models that were initially suggested by the data, thus falling in danger of finding effects that are spurious because random noise is being modeled as structure [41, 42]. In addition, PHRAPL requires estimation of gene trees prior to analysis; so uncertainties in gene tree estimation are not appropriately accounted for, affecting the statistical performance of this method [43].

\section{Species delimitation in the 'gray zone': Caparinia and Dermatophagoides farinae}

The gray zone, an area where conflicting species delimitations are possible, is inherent from the generally continuous nature of the speciation process [44]. However, the typical task of conventional taxonomy is to assign any unknown organism to a species. Considering evidence from analyses based on population genetic theory, STACEY and BPP with realistic priors (small ancestral population and root ages), the two lineages of Caparinia may be considered as two separate, host-specific species, C. tripilis and C. ictonyctis. Similarly, the $7.4-7.8 \%$ of cox 1 sequence divergence (K2P distances), which is well above commonly proposed barcoding thresholds, formally allows these lineages to be considered as separate species (Fig. 4). However, the 7.4-7.8\% cox 1 divergence in the two Caparinia lineages is below our 'conservative' threshold (> 9.5\% or $10.1 \%$, see above). Here we note that these thresholds are based on $>$ species having large effective population sizes <?A3B2 show (Dermatophagoides farinae and Demodex folliculorum), which makes maintaining high genetic diversity in a population more likely [45], but see [46-48]. In 
contrast, the host specific lineages of Caparinia are expected to have very small population sizes, hence, in these populations, speciation may occur much faster than in large populations due to a larger impact of genetic drift $[49,50]$. Furthermore, there are subtle discontinuities in morphological space between the two Caparinia lineages (Fig. 1), and their known native ranges do not overlap, indirectly suggesting that these two populations are indeed genetically isolated, although some gene flow between them still cannot be ruled out. Evidence against the two-species scenario is the presence of a very low synonymous + nonsynonymous divergence in nuclear genes: $0.06,0.09,0.30$ and $0.53 \%$ for $28 S, E F 1-\alpha, S R P 54$, and HSP70, respectively (Additional file 5: Table S2). Except for the latter value, this is substantially below the recently proposed genomic 'gray zone' based on genomic synonymous divergence, $0.5-2 \%$ [51]. Given the above argument we consider the two host-specific lineages as separate species with the caveat that gene flow is possible here. A name for the Caparinia species from the African hosts is already available, Caparinia ictonyctis Lawrence, 1955 stat. res. Previously, this species was considered as a junior synonym of Caparinia tripilis (Michael, 1889) [31].

The American house dust mite, Dermatophagoides farinae, is a system that contrasts with the Caparinia system in having large population sizes. This species is globally distributed and is common in birds' nests, suggesting that it had evolved with birds for a relatively long time, whereas its association with humans is a relatively recent event. Yet, this species has a strong cox1 genetic structure, forming two distinct $\operatorname{cox} 1$ lineages, group 1 and 2, with a maximum divergence of 4.2\% (Additional file 6: Figure S2) or a minimum distance of $9.3 \%$ versus its sibling species, $D$. microceras (Additional file 5: Table S2). These species are reproductively isolated and have distinct differences in the female spermatheca [40]. The strong cox 1 structure observed in $D$. farinae is probably due to past isolation followed by a recent secondary contact; other possible sources of mito-nuclear discordance have been recently reviewed [52]. Cox1-only delimitation approaches all suggested that the traditional scope of $D$. farinae is wrong, and it should be split into two or more species, or even be lumped with $D$. microceras when the prior threshold is larger (Fig. 4). Multispecies coalescent-based methods, BPP (assuming large ancestral population size) and STACEY, recovered $D$. farinae as a single species, in agreement with the traditional taxonomy of this species. This is an example of a clear contrast between results of the two approaches and highlights the importance of using demographics in species delimitation.

\section{Conclusions}

Using DNA-based species delimitation analyses has become a common practice in molecular systematics. Most importantly, the cox1-barcoding approach has become a standard practice of exploring species boundaries in large datasets. We evaluated several standard species delimitation methods and found that they can produce contradictory results, i.e. the 'gray' species delimitation zone, depending on effective population sizes. Populations with large effective sizes can maintain a greater genetic diversity due to their size, which confuses many species delimitation algorithms, resulting in excessive species splitting. This was the case for all species delimitation algorithms, except for STACEY and BPP (only when the population size prior was set appropriately). Particularly, none of the cox1-only barcoding analyses were able to delimit correctly our model species with a large effective population size, the American house dust mite, Dermatophagoides farinae. In contrast, speciation events are more likely in populations with small effective sizes due to genetic drift/random effects. Overall, many species delimitation algorithms, including cox1-only barcoding methods, converge on a single solution here (e.g. two species in the Caparinia dataset). Our study, therefore, highlights the importance of using multilocus datasets and incorporating the knowledge of demographic parameters for DNA-based species delimitation analyses.

\section{Methods}

\section{Material examined}

We nearly exhaustively studied available museum collections and collected new specimens. Type and non-type specimen collection information and host data are given in Additional file 1: Text S1. Live mites (Caparinia from Erinaceus europaeus, ZISP AVB 17-0305-001 and Atelerix albiventris, ZISP AVB 14-0505-004, see Additional file 1: Text S1 for more detail) were removed individually using fine and sharp forceps, preserved in 96\% ethanol for scanning electron microscopy and molecular analysis or mounted in Hoyer's medium [53]. House dust mite datasets (Additional file 2: Table S1) were described previously [54, 55]. For the purpose of this work we consider that census population size and effective population size are highly correlated. Everything else being equal, a species with a small census population size will also have a small effective population size, while a species with a large census population size will likely have a large effective population size (e.g. Dermatophagoides farinae) relative to the rare species (e.g. Caparinia). 
DNA amplification, sequencing and alignment

We sequenced individual specimens of Caparinia from Atelerix albiventris and Erinaceus europaeus for 6 genes: two nuclear ribosomal RNA genes, $18 S$ and $28 S$ rDNA; three nuclear protein-coding genes: elongation factor 1alpha100E $(E F 1-\alpha)$, signal recognition particle protein 54k (SRP54), Hsc70-5 heat shock protein cognate 5 (here abbreviated as HSP70); and one mitochondrial proteincoding gene (cox1). Cox1 was sequenced from 14 specimens for Caparinia ex Atelerix albiventris (all were identical) and 2 specimens of Caparinia tripilis ex Erinaceus europaeus. We used previously published amplification and sequencing protocols [56-59]. To serve as a reference, populations of Dermatophagoides farinae and Dermatophagoides pteronyssinus from both Old and New World populations were sequenced for cox 1 and the nuclear cysteine proteinase-1 preproenzyme gene (CPW2, encoding the major group 1 house dust allergen, abbreviated as Der f1 and Der $p 1$ for the two species, respectively). Primers, amplification, and sequencing of this gene were described previously [54]. GenBank accession numbers are as follows: MG766225MG766259, MG766261-MG766269 (Additional file 2: Table S1). The sequence of $18 S$ of Caparinia from Erinaceus europaeus (GenBank: MG766260) was identified as a gregarine (an endoparasitic protozoan) and, therefore, was excluded from further analyses. Domain D4 of $28 S$ rDNA was also excluded because our standard protocol produced superimposed sequences. rDNA sequences were aligned in Mesquite ver. 3.31 [60] using a previously established secondary structure model [59]; alignment of other loci was unambiguous. Voucher and co-voucher mite specimens are deposited in the University of Michigan Museum of Zoology, Ann Arbor, Michigan under the following accession numbers: Caparinia ictonyctis ex Atelerix albiventris [BMOC 13-0508-003 (AD1647)]; Caparinia tripilis ex Erinaceus europaeus [BMOC 16-0825-012 (AD2034); BMOC 16-0825-013 (AD2035)].

\section{Evaluation of the quality of GenBank sequences}

Sequences deposited in public repositories, such as GenBank, may contain (i) sequencing errors or artifacts (e.g. unnoticed polymerase errors introduced as part of molecular cloning, using low-quality sequence data, or vector/primer sequence contamination); (ii) inaccurate morphology-based identification; (iii) sample contamination or mislabeling. For Pyroglyphidae, we downloaded the available cox1 sequences (GenBank databases: nucleotide, whole genome shotgun contigs, expressed sequence tag) and evaluated their quality using our reference sequences from our specimens carefully identified using morphology. We color-coded our alignment by amino acid transition, and then we looked for unusual amino acid substitutions, stop codons, and frameshifting indels. Maximum likelihood trees with and without the problematic sequences were constructed to see if these sequences could affect phylogenetic inference (Additional file 3: Figure S1, Additional file 4: Alignment S1). For Psoroptidae, we included 12 GenBank sequences, six of which were trimmed to exclude unusual substitutions and frameshifting deletions at the 3 ' end as described previously [57].

\section{Genetic distances}

Following tradition, we use Kimura 2-parameter (K2P) genetic distances [2], but see [61] for criticism. Distances were calculated in PAUP* ver 4.0a (build 158) [62] using the default settings. Uncorrected p-distances were also calculated for comparison; we did not identify extreme K2P values in comparison to uncorrected p-distances, hence we do not report the latter further. We also calculated uncorrected amino acid distances for the same DNA sequences in PAUP. These distances were multiplied by 3 to be compatible with K2P distances on the same plot. To give a morphological context to genetic distance values, taxa were scored as having "clear" (no overlap), "weak" (can mostly be separated morphologically, but with an overlap), or "no" morphological differences from a sister taxon (Additional file 5: Table S2).

\section{Phylogenetic inference}

Substitution models and best partitioning strategies were estimated in PartitionFinder v1.1.1 [63]. The best partitioning scheme contained 7 loci and 4 partitions: $18 \mathrm{~S}$ stem +28 stem $($ TVM $+\mathrm{I}+\mathrm{G}), 18 S$ loop $+28 S$ loop (TIM $+\mathrm{I}+\mathrm{G}), E F 1-\alpha+S R P 54+H S P 70+C P W 2(\mathrm{GTR}+\mathrm{I}+\mathrm{G})$, $\operatorname{cox} 1(\mathrm{GTR}+\mathrm{I}+\mathrm{G})$. An alignment containing 86 individuals classified in 42 morphospecies was used (Additional file 2: Table S1). Phylogenetic relationships were inferred in a Bayesian framework in BEAST 2.4.7, with the clock model set to "Relaxed Clock Log Normal", Yule speciation model, $7.6^{*} 10^{7}$ generations, and a $17 \%$ 'burn-in' as determined by examining ESS values and trace graphs in Tracer. Six independent analyses were run to confirm convergence. A similar maximum likelihood tree was also inferred in RAxML v.8.2.9 [64] (not reported further).

\section{DNA-based species delimitation}

We explored several methods of species delimitation, each making different assumptions (see the Introduction section above): threshold-based ABGD [14]; multispecies coalescent-based; BPP v3.3 [21, 65, 66]; STACEY [22]; PHRAPL [23], and other commonly used species delimitation algorithms, GMYC [24] and PTP [25]. 


\section{GMYC}

This method uses an ultrametric tree and attempts to detect the transition in the tree where the branching pattern switches from being attributed to speciation (one lineage per species) to when it can be attributed to the intra-species coalescent process (multiple lineages per species) [24]. GMYC infers a single cut-off time $\mathrm{T}$ where all nodes above $\mathrm{T}$ represent species; a multithreshold algorithm is available but it is less accurate $[67,68]$. To evaluate sensitivity of the method to different assumptions related to tree priors, we inferred several ultrametric topologies in BEAST using different sets of priors on tree branching and the rate of molecular evolution: a Yule model and a constant clock; a Yule model and a relaxed clock; a coalescent model with constant population size and a constant clock. All BEAST analyses used the GTR $+\mathrm{I}+\mathrm{G}$ model of nucleotide substitution suggested by the program PartitionFinder. Species delimitation analyses were run using the function gmyc of the R package splits v.1.0-19 [69]. For these analyses, we used an 81-taxon, $1238 \mathrm{nt}$ cox1 alignment.

\section{PTP}

This method is similar to GMYC, but it uses branch lengths expressed in terms of nucleotide substitutions, rather than in time units, as required by GMYC. We ran PTP using the webserver (https://cme.h-its.org/exelixis/ web/software/PTP/), which includes both the original maximum likelihood version and the updated Bayesian version (bPTP). Here we used the same dataset as for GMYC analyses (see above) and a phylogenetic tree inferred in RAxML using the GTR $+\mathrm{I}+\mathrm{G}$ model of nucleotide substitution.

\section{$A B G D$}

This program uses a range of prior intraspecific divergences $(\mathrm{P})$ to infer a model-based, one-sided confidence limit for within-species divergence. Then the method detects the barcode gap as the first significant gap beyond this limit and uses it to partition the data. Inference of the limit and gap detection are then recursively applied to previously obtained groups to get finer partitions until no further partitioning occurs [14]. No prior knowledge on species boundaries is required, which is a great advantage of this program. The command-line version of the program was run as follows: "./abgd -a -d 0 -X 0.6 ".fas"; where -a = output all partitions and tree files; $-\mathrm{d} 0=$ computes a matrix of pairwise $\mathrm{K} 2 \mathrm{P}$ distances; $-\mathrm{X}=$ proxy for the minimum gap width, using the default, $\mathrm{X}=1.5$, or any value above 1.1, was impossible because the program did not find more than one partition, so this parameter was varied between $1.1-0.2$ by an increment of -0.1 (10 analyses total); and ".fas = input sequence alignment in fasta format. Other parameter values were defaults. P was varied by the program from 0.001 to 0.100 , which is the default. Because ABGD requires a large training dataset, we employed an 81-taxon 1238 nt cox 1 alignment (same as for GMYC analyses), containing our target taxa (Caparinia, Dermatophagoides farinae), as well as other mites of the two related families, Pyroglyphidae and Psoroptidae, plus outgroups.

\section{BPP}

To evaluate the influence of the ancestral population size $(\theta)$ and root age $(\mathrm{\tau} 0)$ priors on the posterior probabilities of species models, we used three combinations of priors [20]: set1: $\theta \sim \mathrm{G}(1,10) \mathrm{T} 0 \sim \mathrm{G}(1,10)$; set2: $\theta \sim$ $\mathrm{G}(2,1000) \mathrm{T} 0 \sim \mathrm{G}(2,1000)$; and set3: $\theta \sim \mathrm{G}(1,10) \mathrm{\tau} 0 \sim$ $\mathrm{G}(2,1000)$. Other divergence time parameters were assigned the Dirichlet prior (equation 2 in [66]). Set1 assumes large values for both $\theta$ and $\mathrm{t} 0$; Set2 assumes small values for both $\theta$ and $\mathrm{t} 0$; while Set 3 assumes large values for $\theta$ and small values for $\mathrm{t} 0$, favoring conservative models containing fewer species [66]. We used the automatic MCMC fine-tune method for the Dermatophagoides dataset, while for the Caparinia dataset adjustment of finetune variables was necessary. The adjustment was done so that the acceptance proportions are close to 0.3 or lie in the interval $(0.15-0.7)$. For each of the two datasets and each combination of the priors, we conducted two separate analyses: (i) estimating the $\theta$ and $\tau$ parameters (A00: speciesdelimitation $=0$, speciestree $=0$ ) using a tree inferred in RAxML as the guide tree; and (ii) combined species delimitation and species tree inference (A11: speciesdelimitation $=1$, speciestree $=1$ ) with reversible jump (rjMCMC). The heredity scalar was set to 1 (nuclear genes) or 0.25 (mitochondrial genes). All analyses were run for 100,000 generations and a sampling frequency of 1 ; the first $4000 \mathrm{MCMC}$ samples were discarded as 'burn-in'. Marginal likelihoods (Bayes factors) were calculated in BFdriver included in the BPP package; the number of points in the Gauss-Legendre quadrature algorithm for numerical integration was set to $\mathrm{K}=16$. For the Caparinia dataset we used 5 presumably unlinked loci $(18 S+28 S, E F 1-\alpha$, SRP54, HSP7O and cox1) and three putative OTUs: Otodectes cynotis (Ocy), Caparinia ictonyctis from captive Atelerix albiventris (Caic) and Caparinia tripilis from Erinaceus europaeus (Catri). For the Dermatophagoides dataset we used 2 loci ( $\operatorname{cox} 1$ and $C P W 2)$ and four putative OTUs: Dermatophagoides pteronyssinus (DP), $D$. microceras $(\mathrm{DM})$, and $D$. farinae group 1 (DFa) and group $2(\mathrm{DFb})$.

\section{STACEY}

This program is based on multispecies coalescent as implemented in "BEAST [70] but uses an extension of 
this model called the birth-death-collapse model [22]. This model assumes a priori "minimal clusters" of individuals, which can be merged, but not split by the program. There are several priors specific to species delimitation. Most importantly, the Collapse Weight prior provides information about the likely number of species in a delimitation analysis, where values near 1 mean fewer species. In our analyses, the Collapse Weight prior was estimated and set to a uniform distribution $[0,1]$. For the Caparinia dataset, the following models of nucleotide substitution were set for five presumably unlinked loci: TIM (rDNA); TrN (EF1- $\alpha)$; TVM +G (SRP54); TrN+G (HSP70); and TVM+I (cox1). For the two-locus Dermatophagoides dataset, models were as follows: $\mathrm{HKY}+\mathrm{G}(\operatorname{cox} 1)$; TIM+G (CPW2). STACEY was run with the strict clock model; the coalescent parameters were set as suggested in the STACEY manual v1.2.3; MCMC chain length was set to $10^{9}$ sampling every $10^{6}$ generation; 4-7 independent analyses were run to ensure consistency between runs. Runs that converged on a similar distribution were combined. Convergence, mixing, and ESSs were estimated in Tracer v1.6 [71]. For the Caparinia dataset, we evaluated single- and two-species models where Caparinia ictonyctis was either merged with Caparinia tripilis s. str. to form a 'minimal cluster' or these two OTUs were treated separately (see the BPP section above). For the Dermatophagoides dataset, we tested whether Dermatophagoides farinae groups 1 and 2 (DFa, DFb) are one or two species (see the BPP section above). In addition, because of the presence of a large number of individuals, we ran a species discovery analysis, where each individual was treated as a separate 'minimal cluster'. Model comparison was done by using marginal likelihoods (Bayes factors); with standard errors estimated from 16-100 bootstrap replicates in Tracer [71].

\section{PHRAPL}

Because PHRAPL needs at least 3 a priori groups to run, and because the sequence of CTW2 for D. microceras (an outgroup) was not available, we split Dermatophagoides farinae group 2 into two shallow subgroups, $\mathrm{B}$ and $\mathrm{C}$ (Additional file 6: Figure S2). Of these, group $\mathrm{C}$ was a monophyletic, cox1-only lineage (not recovered by $C P W 2$ ). Gene trees were inferred in RAxML, and then they were rooted to mid-point in the $\mathrm{R}$ package phangron [72]. PHRAPL was run with a tip subsampling of 3 tips per 3 populations [popAssignments $<-\operatorname{list}(\mathrm{c}(3,3,3)))$ ], no outgroup (outgroup=FALSE, outgroupPrune=FALSE), and modelRange $=1: 48$ (i.e. all 48 models available in migrationArray); other settings were left at default. We calculated genealogical divergence index (gdi), a composite metric that estimates overall divergence (between 0 and 1) from the combined effects of genetic drift and gene flow, where gdi $=0$ corresponds to panmictic populatuons, while gdi $=1$ corresponds to strong divergence (speciation). It was not possible to run a PHRAPL analysis for Caparinia because there were putative OTUs with fewer than 2 individuals.

For model-based analyses, equivalence of models was established as the following rough rule of thumb [73]: substantial $(\triangle \mathrm{AIC}=0-2)$; weak $(\triangle \mathrm{AIC}=4-7)$; none $(\triangle \mathrm{AIC}>10)$. For model comparison using marginal likelihoods (Bayesian factors, $\mathrm{BF}$ ), the following scale was used [74]: $B F=0-2$ (not worth more than a bare mention); $\mathrm{BF}=2-6$ (positive evidence); $\mathrm{BF}=6-10$ (strong support); and BF > 10 (decisive).

\section{Additional files}

Additional file 1: Text S1. Material studied. (DOCX $19 \mathrm{~kb})$

Additional file 2: Table S1. Taxa, collection data, and GenBank accession numbers. (DOCX $44 \mathrm{~kb}$ )

Additional file 3: Figure S1. Position of low quality sequences of two species of Dermatophagoides on phylogenetic tree. Low quality sequences are identified in nexus file S4 (amino acid color-coded alignment should be viewed in the program Mesquite). (PDF $164 \mathrm{~kb}$ )

Additional file 4: Alignment S1. Alignment of sequences generated in this study and GenBank sequences of Dermatophagoides. Low quality sequences are identified by amino-acid color-coding (should be viewed in the program Mesquite). (NEX $80 \mathrm{~kb}$ )

Additional file 5: Table S2. Within- and among-species genetic distances of 7 loci of 10 putative species. (DOCX $79 \mathrm{~kb}$ )

Additional file 6: Figure S2. Dermatophagoides farinae CO1 and CPW2 gene trees inferred in a Maximum Likelihood framework (RAXML). Important groupings are indicated. (PDF $215 \mathrm{~kb}$ )

Additional file 7: Table S3. Summary of species delimitation analyses. (XLSX $22 \mathrm{~kb}$ )

Additional file 8: Figure S3. Similarity matrix of STACEY species discovery analysis of the Dermatophagoides dataset. (PDF $138 \mathrm{~kb}$ )

Additional file 9: Table S4. Summary of PHRAPL analyses of the Dermatophagoides dataset. (XLSX $90 \mathrm{~kb}$ )

Additional file 10: Alignment S2. Nexus datamatrix of aligned sequences of seven loci used to infer species tree (Fig. 4). Original contiguous alignments as well as final partitions (identified by the suffix _noee») used in analyses are given. The file should be viewed in the program Mesquite. (NEX $7275 \mathrm{~kb}$ )

\section{Abbreviations}

G: Gamma parameter; I: Invariant site parameter; 18S: Small subunit ribosomal RNA gene; 28S: Large subunit ribosomal RNA gene; rDNA: Ribosomal DNA; ABGD: Automatic Barcode Gap Discovery (species delimitation algorithm); AIC: Akaike information criterion; BEAST: Bayesian Evolutionary Analysis Sampling Trees (phylogenetic tree inference program); BF: Bayes Factor; BPP: Bayesian Phylogenetics and Phylogeography (species delimitation algorithm); bPTP: Bayesian Poisson Tree Processes model (species delimitation algorithm); cox1: Cytochrome $\mathrm{c}$ oxidase subunit 1 gene; CPW2: cysteine protease precursor (this locus has specific abbreviations for each species; Der p1: Group 1 allergen preproenzyme for Dermatophagoides pteronyssinus; Der f 1: Group 1 allergen preproenzyme for D. farinae; EF1a: Elongation factor 1alpha100E gene; ESS: Effective sample size of MCMC (Markov chain Monte Carlo); gdi: Genealogical divergence index; GMYC: Generalized Mixed Yule Coalescent (species delimitation algorithm); GTR: General time reversible model of nucleotide evolution; HKY: Hasegawa, Kishino \& Yano, 1985 model of nucleotide evolution; HSP70: heat-shock protein cognate 5 gene; K2P: Kimura 2-parameter); OTU: Operational 
taxonomic unit; PAUP*: Phylogenetic Analysis Using Parsimony and Other Methods (phylogenetic tree inference program); PHRAPL: Phylogeography using Approximate Likelihood (species delimitation algorithm); PTP: Poisson Tree Processes model (species delimitation algorithm); RAxML: Randomized Accelerated Maximum Likelihood (phylogenetic tree inference program); rjMCMC: reversible jump Markov chain Monte Carlo; SRP54: Signal recognition particle protein 54k gene; STACEY: Species Tree And Classification Estimation, Yarely (species delimitation and phylogeny estimation algorithm); TIM: transitional model of nucleotide evolution TrN: Tamura \& Nei model of nucleotide evolution; TVM: transversional model of nucleotide evolution; $X$ : barcode gap width proxy (applies to ABGD); $\Delta$ : change of a value; $\theta$ : ancestral population size; $\tau$ : root age

\section{Acknowledgements}

We thank Drs Patrick Grootaert (Institut Royal des Sciences Naturelles de Belgique, Brussels, Belgium) and Didier Van den Spiegel (Musee Royal de Afrique Centrale, Tervuren, Belgium) who loaned specimens of Caparinia spp. deposited in A. Fain's collections. Dr Anne Baker (Natural History Museum, London, England) confirmed the presence of the Caparinia tripilis holotype in NHM and photograped the slide label for us. Da-Hee Kim (National University, Gwangju, South Korea) sent us Caparinia specimens from the African hedgehog. We are grateful to Barry OConnor (University of Michigan) who gave useful comments on the manuscript. The molecular work of this study was conducted in the Genomic Diversity Laboratory of the University of Michigan Museum of Zoology.

\section{Funding}

This research was supported by the Polish National Science Centre (Grant No. NCN 2014/15/B/NZ8/00208) to MS, by the Russian Foundation for Basic Research (grant No. 15-29-02533 to AVB and 18-04-01092A to PBK), and by the Russian Science Foundation (grant 16-14-10109) to A. Khaustov.

\section{Availability of data and materials}

All data analysed during this study are included in this published article and its additional files. New sequences generated in this work were deposited in the GenBank database under the accession numbers MG766225-MG766259 and MG766261-MG766269.

\section{Authors' contributions}

All authors designed experiments, wrote manuscript. AVB and PBK carried out data analyses and sample collection. PBK performed DNA sequencing. All authors read and approved the final manuscript.

\section{Ethics approval and consent to participate}

Not applicable.

\section{Consent for publication}

Not applicable.

\section{Competing interests}

The authors declare that they have no competing interests.

\section{Publisher's Note}

Springer Nature remains neutral with regard to jurisdictional claims in published maps and institutional affiliations.

\footnotetext{
Author details

'Department of Ecology and Evolutionary Biology, Museum of Zoology, University of Michigan, 3600 Varsity Drive, Ann Arbor, Michigan 48108, USA ${ }^{2}$ Tyumen State University, 10 Semakova Str, 625003 Tyumen, Russia. ${ }^{3}$ Department of Animal Morphology, Faculty of Biology, Adam Mickiewicz University, Umultowska 89, 60-614 Poznan, Poland. ${ }^{4}$ Zoological Institute, Russian Academy of Sciences, Universitetskaya emb. 1, 199034 St Petersburg, Russia.
}

Received: 29 October 2018 Accepted: 28 November 2018 Published online: 05 January 2019

\section{References}

1. Hebert PDN, Ratnasingham S, deWaard JR. Barcoding animal life: cytochrome $\mathrm{C}$ oxidase subunit 1 divergences among closely related species. Proc R Soc B-Biol Sci. 2003;270:S96-9.

2. Hebert PDN, Cywinska A, Ball SL, DeWaard JR. Biological identifications through DNA barcodes. Proc R Soc B-Biol Sci. 2003;270:313-21.

3. Ratnasingham S, Hebert PDN. BOLD: The Barcode of Life Data System (http://www.barcodinglife.org). Mol Ecol Notes. 2007;7:355-64.

4. Schoch $C L$, Seifert KA, Huhndorf S, Robert V, Spouge $J$, Levesque CA, et al. Nuclear ribosomal internal transcribed spacer (ITS) region as a universal DNA barcode marker for Fungi. Proc Natl Acad Sci USA. 2012;109:6241-6.

5. Chase MW, Soltis DE, Olmstead RG, Morgan D, Les DH, Mishler BD, et al. Phylogenetics of seed plants - an analysis of nucleotide-sequences from the plastid gene Rbcl. Ann Mo Bot Gard. 1993;80:528-80.

6. Hebert PDN, Penton EH, Burns JM, Janzen DH, Hallwachs W. Ten species in one: DNA barcoding reveals cryptic species in the Neotropical skipper butterfly Astraptes fulgerator. Proc Natl Acad Sci USA. 2004;101:14812-7.

7. Collins RA, Cruickshank RH. Known knowns, known unknowns, unknown unknowns and unknown knowns in DNA barcoding: a Comment on Dowton et al. Syst Biol. 2014;63:1005-9.

8. Yang ZH, Rannala B. Bayesian species identification under the multispecies coalescent provides significant improvements to DNA barcoding analyses. Mol Ecol. 2017;26:3028-36.

9. Meier R, Shiyang K, Vaidya G, Ng PKL. DNA barcoding and taxonomy in Diptera: a tale of high intraspecific variability and low identification success. Syst Biol. 2006;55:715-28.

10. Liu JN, Jiang JM, Song SL, Tornabene L, Chabarria R, Naylor GJP, Li CH. Multilocus DNA barcoding - species identification with multilocus data. Sci Rep. 2017;7:16601.

11. Dowton M, Meiklejohn K, Cameron SL, Wallman J. A preliminary framework for DNA barcoding, incorporating the multispecies coalescent. Syst Biol. 2014;63:639-44.

12. Smith MA, Fisher BL, Hebert PDN. DNA barcoding for effective biodiversity assessment of a hyperdiverse arthropod group: the ants of Madagascar. Philos Trans R Soc B-Biol Sci. 2005;360:1825-34.

13. Rossini BC, Oliveira CAM, de Melo FAG, Bertaco VD, de Astarloa JMD, Rosso J, et al. Highlighting Astyanax species diversity through DNA barcoding. PLoS One. 2016;11:e0167203.

14. Puillandre N, Lambert A, Brouillet S, Achaz G. ABGD, Automatic Barcode Gap Discovery for primary species delimitation. Mol Ecol. 2012;21:1864-77.

15. Palopoli MF, Fergus DJ, Minot S, Pei DT, Simison WB, Fernandez-Silva I, et al. Global divergence of the human follicle mite Demodex folliculorum: persistent associations between host ancestry and mite lineages. Proc Natl Acad Sci USA. 2015;112:15958-63.

16. Wiemers $M$, Fiedler K. Does the DNA barcoding gap exist? - a case study in blue butterflies (Lepidoptera: Lycaenidae). Front Zool. 2007;4:1-16.

17. Williams ST, Reid DG. Speciation and diversity on tropical rocky shores: a global phylogeny of snails of the genus Echinolittorina. Evolution. 2004:58:2227-51.

18. Murillo P, Klimov PB, Hubert J, OConnor BM. Investigating species boundaries using DNA and morphology in the mite Tyrophagus curvipenis (Acari: Acaridae), an emerging invasive pest, with a molecular phylogeny of the genus Tyrophagus. Exp Appl Acarol. 2018;75:167-89.

19. Dellicour S, Flot JF. Delimiting species-poor data sets using single molecular markers: a study of barcode gaps, haplowebs and GMYC. Syst Biol. 2015;64:900-8.

20. Leache AD, Fujita MK. Bayesian species delimitation in West African forest geckos (Hemidactylus fasciatus). Proc R Soc B-Biol Sci. 2010;277:3071-7.

21. Yang ZH. The BPP program for species tree estimation and species delimitation. Curr Zool. 2015;61:854-65.

22. Jones $\mathrm{G}$. Algorithmic improvements to species delimitation and phylogeny estimation under the multispecies coalescent. J Math Biol. 2017;74:447-67.

23. Jackson ND, Carstens BC, Morales AE, O'Meara BC. Species delimitation with gene flow. Syst Biol. 2017;66:799-812.

24. Pons J, Barraclough TG, Gomez-Zurita J, Cardoso A, Duran DP, Hazell S, et al. Sequence-based species delimitation for the DNA taxonomy of undescribed insects. Syst Biol. 2006;55:595-609. 
25. Zhang JJ, Kapli P, Pavlidis P. Stamatakis A. A general species delimitation method with applications to phylogenetic placements. Bioinformatics. 2013; 29:2869-76.

26. Mullen GR, OConnor BM. Mites (Acari). In: Mullen GR, Durden LA, editors. Medical and Veterinary Entomology. London: Elsevier; 2002. p. 449-516.

27. Demkowska-Kutrzepa M, Tomczuk K, Studzinska M, Szczepaniak K. Caparinia tripilis in African hedgehog (Atelerix albiventris). Vet Dermatol. 2015;26:73-5.

28. Moreira A, Troyo A, Calderon-Arguedas O. First report of acariasis by Caparinia tripilis in African hedgehogs, (Atelerix albiventris), in Costa Rica. Rev Bras Parasitol Vet. 2013;22:155-8.

29. Romero C, Waisburd GS, Pineda J, Heredia R, Yarto E, Cordero AM. Fluralaner as a single dose oral treatment for Caparinia tripilis in a pygmy African hedgehog. Vet Dermatol. 2017;28:622-e152.

30. Kim DH, Oh DS, Ahn KS, Shin SS. An outbreak of Caparinia tripilis in a Colony of African pygmy hedgehogs (Atelerix albiventris) from Korea. Kor J Parasitol. 2012;50:151-6.

31. Fain A. Nouveaux taxa dans les Psoroptinae hypothese sur l'origine de ce groupe (Acarina, Sarcoptiformes, Psoroptidae). Acta Zool Pathol Antverp. 1975;61:57-84

32. Lawrence RF. A new mange-mite from the Cape polecat. Ann Trop Med Parasitol. 1955:49:54-62.

33. Candek K, Kuntner M. DNA barcoding gap: reliable species identification over morphological and geographical scales. Mol Ecol Res. 2015;15:268-77.

34. Doña J, Diaz-Real J, Mironov S, Bazaga P, Serrano D, Jovani R. DNA barcoding and minibarcoding as a powerful tool for feather mite studies. Mol Ecol Res. 2015;15:1216-25.

35. Zahler M, Essig A, Gothe R, Rinder H. Genetic evidence suggests that Psoroptes isolates of different phenotypes, hosts and geographic origins are conspecific. Int J Parasitol. 1998;28:1713-9.

36. Pegler KR, Evans L, Stevens JR, Wall R. Morphological and molecular comparison of host-derived populations of parasitic Psoroptes mites. Med Vet Entomol. 2005;19:392-403.

37. Ramey RR, Kelley ST, Boyce WM, Farrell BD. Phylogeny and host specificity of psoroptic mange mites (Acarina: Psoroptidae) as indicated by ITS sequence data. J Med Entomol. 2000;37:791-6.

38. Amer S, Abd El Wahab T, Metwaly A, Feng YY, Xiao LH. Morphologic and genotypic characterization of Psoroptes mites from water buffaloes in Egypt. PLoS One. 2015;10:e0141554

39. Bates PG. Inter- and intra-specific variation within the genus Psoroptes (Acari: Psoroptidae). Vet Parasitol. 1999;83:201-17.

40. Griffiths DA, Cunnington AM. Dermatophagoides microceras sp. n.: a description and comparison with its sibling species, D. farinae Hughes. J Stored Prod Res. 1971;7:1-14

41. Moore BR, Donoghue MJ. A Bayesian approach for evaluating the impact of historical events on rates of diversification. Proc Natl Acad Sci USA. 2009; 106:4307-12.

42. Mosteller F. A k-sample slippage test for an extreme population. Ann Math Stat. 1948;19:58-65.

43. $\mathrm{Xu} \mathrm{B}$, Yang $\mathrm{ZH}$. Challenges in species tree estimation under the multispecies coalescent model. Genetics. 2016;204:1353-68

44. De Queiroz K. Species concepts and species delimitation. Syst Biol. 2007;56:879-86

45. Charlesworth B. Fundamental concepts in genetics: effective population size and patterns of molecular evolution and variation. Nat Rev Genet. 2009;10:195-205.

46. Bazin E, Glémin S, Galtier N. Population size does not influence mitochondrial genetic diversity in animals. Science. 2006;312:570.

47. Ellegren $\mathrm{H}$. Is genetic diversity really higher in large populations? J Biol. 2009;8:41.

48. Evolution E-WA. Size does not matter for mitochondrial DNA. Science. 2006; 312:537-8.

49. Grossenbacher DL, Veloz SD, Sexton JP. Niche and range size patterns suggest that speciation begins in small, ecologically diverged populations in North American monkeyflowers (Mimulus spp.). Evolution. 2014;68:1270-80.

50. Nei M, Maruyama T, Wu Cl. Models of evolution of reproductive isolation. Genetics. 1983;103:557-79.

51. Roux C, Fraisse C, Romiguier J, Anciaux Y, Galtier N, Bierne N. Shedding light on the grey zone of speciation along a continuum of genomic divergence. PLoS Biol. 2016;14:e0141554.

52. Toews DPL, Brelsford A. The biogeography of mitochondrial and nuclear discordance in animals. Mol Ecol. 2012;21:3907-30.
53. Evans GO. Principles of Acarology. London: CAB International; 1992.

54. Shafique RH, Klimov PB, Inam M, Chaudhary FR, OConnor BM. Group 1 allergen genes in two species of house dust mites, Dermatophagoides farinae and D. pteronyssinus (Acari: Pyroglyphidae): direct sequencing, characterization and polymorphism. PLoS One. 2014;9:e114636.

55. Klimov PB, Bochkov AV, OConnor BM. Phylogenetic position of the house dust mite subfamily Guatemalichinae (Acariformes: Pyroglyphidae) based on integrated molecular and morphological analyses and different measures of support. Cladistics. 2016:32:261-75.

56. Klimov PB, OConnor BM. Is permanent parasitism reversible? - Critical evidence from early evolution of house dust mites. Syst Biol. 2013;62:411-23.

57. Bochkov AV, Klimov PB, Hestvik G, Saveljev AP. Integrated Bayesian species delimitation and morphological diagnostics of chorioptic mange mites (Acariformes: Psoroptidae: Chorioptes). Parasitol Res. 2014;113:2603-27.

58. Knowles L, Klimov PB. Estimating phylogenetic relationships despite discordant gene trees across loci: the species tree of a diverse species group of feather mites (Acari: Proctophyllodidae). Parasitology. 2011;138: 1750-9.

59. Klimov PB, OConnor BM. Origin and higher-level relationships of psoroptidian mites (Acari: Astigmata: Psoroptidia): evidence from three nuclear genes. Mol Phylogenet Evol. 2008;47:1135-56.

60. Maddison WP, Maddison DR. Mesquite: a modular system for evolutionary analysis. Version 3.10. 2016. http://mesquiteproject.org.

61. Srivathsan A, Meier R. On the inappropriate use of Kimura-2-parameter (K2P) divergences in the DNA-barcoding literature. Cladistics. 2012;28:190-4.

62. Swofford DL. PAUP*. Phylogenetic Analysis Using Parsimony (*and other methods). Version 4.0a150. Distributed by the author; 2016.

63. Lanfear R, Calcott B, Ho SY, Guindon S. Partitionfinder: combined selection of partitioning schemes and substitution models for phylogenetic analyses. Mol Biol Evol. 2012;29:1695-701.

64. Stamatakis A. RAxML version 8: a tool for phylogenetic analysis and postanalysis of large phylogenies. Bioinformatics. 2014;30:1312-3.

65. Rannala B, Yang Z. Bayes estimation of species divergence times and ancestral population sizes using DNA sequences from multiple loci. Genetics. 2003;164:1645-56.

66. Yang ZH, Rannala B. Bayesian species delimitation using multilocus sequence data. Proc Natl Acad Sci USA. 2010;107:9264-9.

67. Fujita MK, Leache AD, Burbrink FT, McGuire JA, Moritz C. Coalescent-based species delimitation in an integrative taxonomy. Trends Ecol Evol. 2012;27:480-8.

68. Blair C, Bryson RW. Cryptic diversity and discordance in single-locus species delimitation methods within horned lizards (Phrynosomatidae: Phrynosoma). Mol Ecol Res. 2017;17:1168-82.

69. Ezard T, Fujisawa T, Barraclough T. splits: SPecies' LImits by Threshold Statistics. R package version 1.0-19/r52. 2017. https://R-Forge.R-project.org/ projects/splits/.

70. Heled J, Drummond AJ. Bayesian inference of species trees from multilocus data. Mol Biol Evol. 2010;27:570-80.

71. Rambaut A, Drummond AJ. Tracer v1.6. 2009. Available from http:/tree.bio. ed.ac.uk/software/tracer/.

72. Schliep KP. phangorn: phylogenetic analysis in R. Bioinformatics. 2011:27:592-3.

73. Burnham KP, Model Selection ADR. Multimodel Inference: A Practical Information-Theoretic Approach. 2nd ed. New York: Springer-Verlag; 2002.

74. Kass RE, Raftery AE. Bayes factors. J Am Stat Assoc. 1995;90:773-95.

Ready to submit your research? Choose BMC and benefit from:

- fast, convenient online submission

- thorough peer review by experienced researchers in your field

- rapid publication on acceptance

- support for research data, including large and complex data types

- gold Open Access which fosters wider collaboration and increased citations

- maximum visibility for your research: over $100 \mathrm{M}$ website views per year

At $\mathrm{BMC}$, research is always in progress.

Learn more biomedcentral.com/submissions 